\title{
Comparison of Experimental Investigation of Deflection of the Sandwich Com- posite Beam by Optic-fibre Gauge with Theoretical Models
}

Dita Jiroutova

Klokner Institute, Czech Technical University in Prague. Šolínova 7, 16608 Prague 6. Czech Republic. E-mail: Dita.Jiroutova@crut.cz

The aim of the article is to compare the experimental investigation of deflection of the sandwich composite beam with theoretical models. The experimental test specimens were composed of three layers. Skins were made using epoxy-resin-impregnated glass laminates with plain weave. The light weight foam Divinycell H100 was used as sandwich core. The three-point bending test was carried out. Fibre-optic strain gauges, SOFO SMARTape Compact deformation sensors, were used for determining the deflection of the sandwich composite structure. Experimentally obtained data were used for comparison with theoretical models - sandwich theory with the transverse shear, sandwich theory without the transverse shear, laminate theory with the transverse shear and laminate theory without the transverse shear.

Keywords: Deflection, Sandwich Composite Beam, Fibre-optic Gauge, Classical Lamination Theory, Sandwich Theory

\section{Introduction}

Due to its advantageous properties like stiffness, high impact strength, corrosion resistance, low thermal conductivity and low acoustic conductivity, the sandwich composite structures are commonly used in civil engineering, space, marine and automobile industry etc. [1 - 4]. The sandwich composite structures are composed of two main parts - the skin and the core. The skin is thin layer with a very high stiffness. The main function of the skin is to carry tension, compression and bending moments which affect the sandwich composite structure. The skin is bonded to the sandwich core. The core of the sandwich structure is thick and lightweight. The function of the core is to carry transverse shear applied to the structure. So it means, sandwich composite structures have high shear stiffness to the weight ratio and high tensile strength to the weight ratio. The presence of one or more types of materials having different mechanical properties in each layer causes abrupt change of the properties between layers. Abrupt change of properties between the layers causes interlaminar stress which can lead to destruction of the structure [5]. A good knowledge of the sandwich composite structure behaviour is important for efficient manufacture techniques, long-term prediction of behaviour and also for economics and designing the sandwich composite structures.

Nowadays, a lot of theories describing behaviour of the sandwich composite structures exist. The simple two-dimensional model called the classical laminated plate theory is based on Kirchhoff model and is used in case of the isotropic plates. The classical laminated theory can be used for thin plates $[6,7]$. This theory ignores deformation due to the transverse shear and reflects that the normal load remains straight to the core [8]. The classical plate theory is based on displacement analysis and it satisfies requirements of the continuity of displacement along the thickness [9]. The first-order shear deformation theory is proposed by Mindlin and Reissner. This theory assumes constant transverse shear stress, but needs using shear correction factors [10]. The first-order shear deformation theory is required in case of strong anisotropy of mechanical properties or for thick sandwich plates [11]. The higher-order shear deformation theory is more accurate method than the classical laminated theory and first-order shear deformation theory. This theory assumes higher variations of surface parallel displacements through the thickness of the laminates [7]. Comparison of these theories with experimental results can be found in literature, e.g. [12], [13] etc.

The aim of the article is the comparison of experimental investigation of deflection of the sandwich composite beam with theoretical models. The laminate theory with transverse shear, the laminate theory without transverse shear, the sandwich theory with transverse shear and the sandwich theory without transverse shear were used for comparison with experimental results. This paper loosely follows the article [14] dealing with methodology of measurement.

\section{Theoretical models}

The three-layered sandwich composite was used in the experimental part. The same type of the sandwich composite was considered in theory part. Considered 
sandwich composite with direction $x, y$ and $z$ is mentioned in Fig. 1.

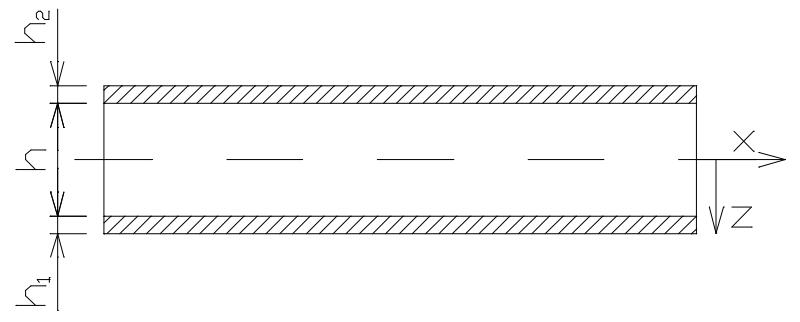

Fig. 1 Sandwich composite structure

\subsection{Laminate theory without transverse shear}

Classical laminate theory does not include transverse shear and describes behaviour of laminate structure. The laminate structure consists of $n$ layers. This theory assumes that

- $\quad$ each layer of laminate structure is orthotropic and quasihomogenous,

- the transverse size of the laminate is small compared to the other two dimensions of the laminate. This theory assumes two-directional stress field,

- displacements of all points are really small,

- the joint between layers is faultless and really thin. This causes continuous displacements. Displacements in transverse direction change linear,

- the transverse deformation is neglected,

- linear dependence between stress and deformation.

Then the constitutive equation of the symmetric laminate in the case of bending without transverse shear can be expressed by equation [15]

$$
\left[\begin{array}{l}
M_{x} \\
M_{y} \\
M_{x y}
\end{array}\right]=\left[\begin{array}{lll}
D_{11} & D_{12} & D_{16} \\
D_{12} & D_{22} & D_{26} \\
D_{16} & D_{26} & D_{66}
\end{array}\right]\left[\begin{array}{c}
\kappa_{x} \\
\kappa_{y} \\
\kappa_{x y}
\end{array}\right]
$$

where $D_{i j}$ are the elements of the bending stiffness matrix. The elements of the matrix $D_{i j}$ are calculated for each layer and consider if the layer is tensioned or compressed. $M_{x}$ and $M_{y}$ are the bending moments, $M_{x y}$ is the twisting moment. $\boldsymbol{\kappa}_{x}, \boldsymbol{\kappa}_{y}$ and $\boldsymbol{\kappa}_{x y}$ are the curvatures.

Then the deflection at the middle of the span of the laminate beam $y_{\max }$ loaded by three-point bending can be determined by equation

$$
y_{\max }=\frac{F L^{3}}{48 b} D_{11}^{*}
$$

where $F, L, b$ and $D^{*} 11$ are the force, beam length between supports, beam width, and elements of the inverse matrix $D^{*}$ to the bending stiffness matrix $D$, respectively. The elements of the bending stiffness matrix $D$ are calculated from equation

$$
D_{i j}=\frac{1}{3} \sum_{k=1}^{n}\left(C_{i j}\right)_{k}\left(h_{k}^{3}-h_{k-1}^{3}\right)
$$

where $C_{i j}$ are the elements of the stiffness matrix and these elements are function of Poisson number and Young's modulus of elasticity, $k$ is the layer $(k=$ $1,2, \ldots n)$ and $h$ is the layer thickness.

\subsection{Laminate theory with transverse shear}

This laminate theory includes transverse shear. This theory assumes that

- the core carries shear as well as bending,

- the core is isotropic,

- the core is considered as one layer,

- Kirchoff hypothesis is not valid.

The constitutive equation of the symmetric laminate in the case of bending with transverse shear can be expressed by [15]

$$
\left[\begin{array}{c}
M_{x} \\
M_{y} \\
M_{x y} \\
Q_{y} \\
Q_{x}
\end{array}\right]=\left[\begin{array}{ccccc}
D_{11} & D_{12} & D_{16} & 0 & 0 \\
D_{12} & D_{22} & D_{26} & 0 & 0 \\
D_{16} & D_{26} & D_{66} & 0 & 0 \\
0 & 0 & 0 & F_{44} & F_{45} \\
0 & 0 & 0 & F_{45} & F_{55}
\end{array}\right]\left[\begin{array}{c}
\kappa_{x} \\
\kappa_{y} \\
\kappa_{x y} \\
\gamma_{y z}^{0} \\
\gamma_{x z}^{0}
\end{array}\right]
$$

where $D_{i j}$ are the elements of the bending stiffness matrix. The elements of the matrix $D_{i j}$ are calculated for each layer and consider if the layer is tensioned or compressed. $F_{i j}$ are the elements of the shear stiffness matrix. $M_{x}$ and $M_{y}$ are bending moments, $M_{x y}$ is twisting moment. $Q_{x}$ and $Q_{y}$ are transverse shear, $\kappa_{x}, \kappa_{y}$ and $\kappa_{x y}$ are curvatures, $\gamma_{y z}$ and $\gamma_{x z}$ are slopes.

The deflection in the middle of the span of the laminate beam $y_{\max }$ loaded by three-point bending is determined from equation

$$
y_{\max }=\frac{F L^{3}}{48 b} D_{11}^{*}+\frac{F L}{4 b} F_{55}^{*}
$$

where $F, L b, D^{*}{ }_{11}$, and $F^{*}{ }_{55}$ are the force, beam length between supports, beam width, elements of the inverse matrix $D^{*}$ to the matrix $D$, and elements of the inverse matrix $F^{*}$ to the shear stiffness matrix $F$, respectively. The elements of the shear stiffness matrix $F$ are calculated from equation

$$
F_{i j}=\sum_{k=1}^{n}\left(C_{i j}\right)_{k}\left(h_{k}-h_{k-1}\right)
$$

where $C_{i j}$ are the elements of the stiffness matrix and these elements are function of Poisson number and Young's modulus of elasticity, $k$ is the layer $(k=$ $1,2, \ldots n)$ and $b$ is the layer thickness.

\subsection{Sandwich theory without transverse shear}

This sandwich theory does not include the transverse shear. This theory assumes that

- deformations are small,

- Hook's law is valid,

- the core thickness is greater than thickness of 
the skins,

- displacements of the core in direction $x$ and $y$ changes linearly in the transverse direction,

- displacements of the skin core in direction $x$ and $y$ are constant through the sandwich thickness,

- the transverse displacement is independent on coordinate $z$ and it can be assumed that the deformation in direction $z$ is equal to zero,

- the effect of the shear stress is neglected.

The constitutive equation of the symmetric sandwich composite structure in the case of bending without transverse shear can be expressed by equation (1). The elements of the matrix $D_{i j}$ are calculated for each layer and does not consider if the layer is tensioned or compressed. The deflection in the middle of the span of the sandwich composite beam $y_{\max }$ loaded by threepoint bending is determined from equation (2).

\subsection{Sandwich theory with transverse shear}

This sandwich theory includes the transverse shear. This theory assumes that

- deformations are small,

- Hook's law is valid,

- the core thickness is greater than thickness of the skins,

- displacements of the core in direction $\mathrm{x}$ and $\mathrm{y}$ changes linearly in the transverse direction,

- displacements of the skins core in direction $\mathrm{x}$ and $y$ are constant through the sandwich thickness,

- the transverse displacement is independent on coordinate $z$ and it can be assumed that the deformation in direction $z$ is equal to zero,

- the core carries only transverse shear stress,

- skin carries only bending.

The constitutive equation of the symmetric sandwich composite structure in the case of bending with transverse shear can be expressed by equation (4) where the elements of the matrix $D_{i j}$ are calculated for each layer and it does not consider if the layer is tensioned or compressed. The deflection in the middle of the sandwich composite beam span $y_{\text {max }}$ loaded by three-point bending is determined from equation (5).

\section{Sandwich composite structure}

Five sandwich composite test specimens were used for experiments. The length of test specimen was 700 $\mathrm{mm}$, width $75 \mathrm{~mm}$ and thickness approximately 22 $\mathrm{mm}$. The experimental sandwich composite test specimen was composed of three layers. Skin was made from epoxy-resin-impregnated glass laminate with plain weave with weight $600 \mathrm{~g} / \mathrm{m}^{2}$. The thickness of the skin is $0.8 \mathrm{~mm}$. The light weight foam Divinycell H100, manufactured by DIAB GROUP, was used as the sandwich core. The foam core Divinycell H100 is a combination of polyurea and PVC. The thickness of the foam core was $20 \mathrm{~mm}$ and density $100 \mathrm{~kg} / \mathrm{m}^{3}$. Two SOFO® SMARTape Compact deformation sensors were placed in every sandwich composite test specimen. The fibre-optic extensometers were embedded between skin and light weight foam core. One of them was installed between upper skin and foam core and the second extensometer was installed between bottom skin and sandwich foam core. Two components toughened with methacrylate adhesive system Araldite ${ }^{\circledR} 2021$ were used for installation of fibre-optic sensors and for bonding skins to the sandwich foam core. Each SOFO ${ }^{\circledR}$ SMARTape Compact deformation sensor was installed in the middle of sandwich composite test specimen width. The diagrammatic scheme of the tested sandwich composite structure is shown in Fig. 2. Photo of the tested sandwich beam with embedded SOFO® SMARTape Compact deformation sensors is shown in Fig. 3.

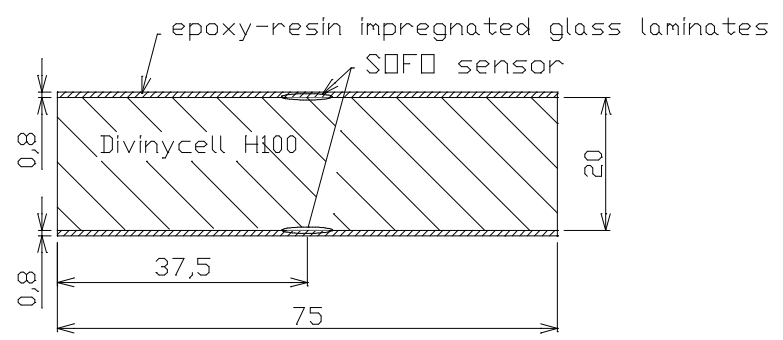

Fig. 2 Diagrammatic scheme of the tested sandwich composite structure with embedded SOFO ${ }^{\circledR}$ SMARTape Compact deformation sensor

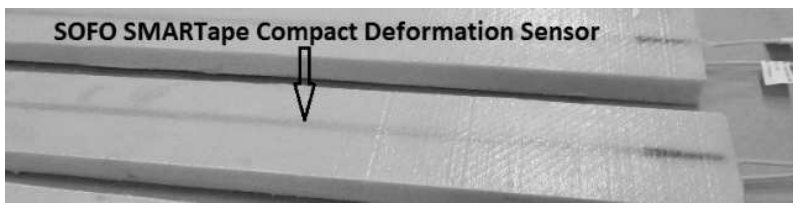

Fig. 3 Tested sandwich composite structure with embedded SOFO® SMARTape Compact deformation sensor

\section{Experiments}

\subsection{Sandwich beam}

Three-point bending test was carried out. The length of the sandwich composite test specimen was $700 \mathrm{~mm}$ and support distance $600 \mathrm{~mm}$. The bending load was applied only by means of own weight of the steel cylindrical supports. Cylindrical support diameter was $50 \mathrm{~mm}$ and length $80 \mathrm{~mm}$. Weight of one support was approx. $1.2 \mathrm{~kg}$. It means load induced by one support was approx. $12 \mathrm{~N}$. 
The SOFO® SMARTape Compact deformation sensors with measuring length $600 \mathrm{~mm}$ were used for measuring the sandwich composite deformation. This sensor is embeddable between composite layers due to its extremely small cross-section. These sensors are insensitive to temperature variations, corrosion, vibrations, and immune to electromagnetic field. The measurement range is $1.5 \%$ of the length in shortening and $1.5 \%$ of the length in elongation. The resolution is $0.002 \mathrm{~mm}$. The SOFO ${ }^{\circledR}$ SMARTape Compact deformation sensors are transducers that transform a strain variation into a change in the path unbalance between two optical fibres that are measured with the SOFO reading unit [16]. The SOFO measuring system for static quantities with SBD ver. 6.3.53 software from SMARTEC S.A. was used for recording the measurement readings. Arrangement of the experiments is shown in Fig. 4.

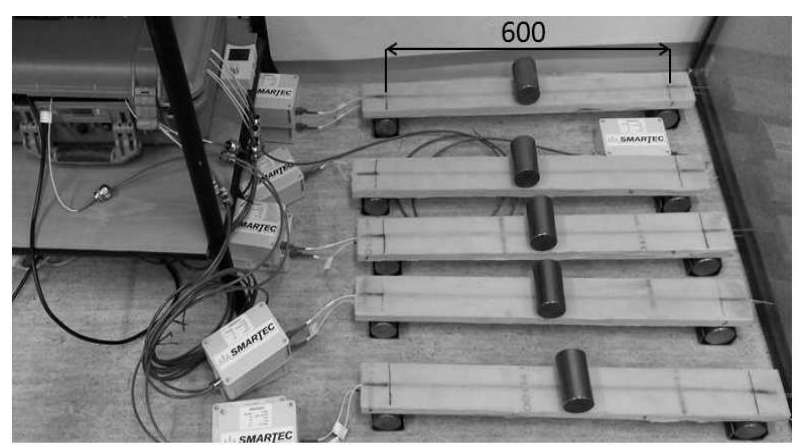

Fig. 4 Arrangement of the experiments [17]

The three-point bending test took three months. This period was chosen due to the suppression of the aging effects. Each test specimen was load in different way. Test specimens loading is shown in Fig. 5.

Deformations of the sandwich composite beam were measured by the SOFO ${ }^{\circledR}$ SMARTape Compact deformation sensors. The deformations were measured constantly during three months. Measurement were recorded every hour by the SOFO measuring system for static quantities. Deflections were determined for every record value of the deformations obtained from upper and lower sensor. Deflection was calculated from equation of the deflection line. Finally, the average value of deflection for every load was calculated and this calculation was based on values of deflection obtained from upper and lower sensors.

The three-point bending load was carried out in laboratory. Temperature and air humidity in laboratory were also measured during three-point bending test. Temperature in the laboratory was held at $(20 \pm 1){ }^{\circ} \mathrm{C}$ and relative air humidity was $(41 \pm 5) \%$.

Obtained deformations measured by fibre-optic SOFO ${ }^{\circledR}$ SMARTape Compact deformation sensors bonded between upper skin and core (upper sensor) and bottom skin and core (lower sensor) are for illustration mentioned in Fig. 6.

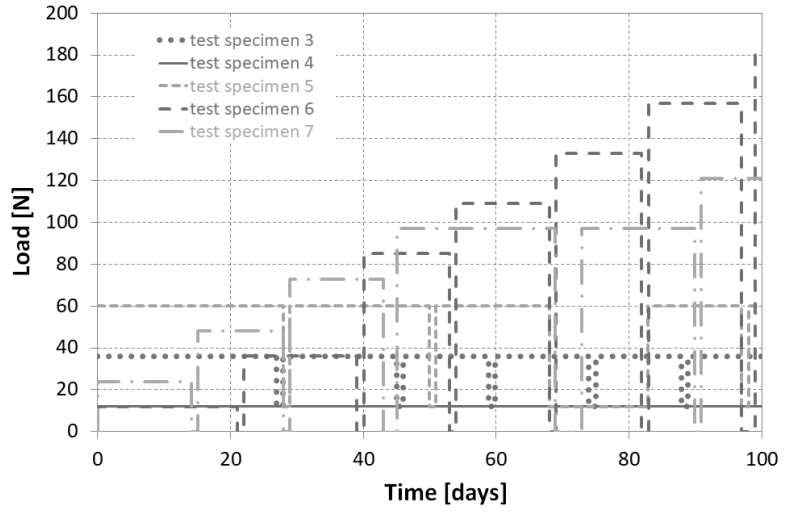

Fig. 5 Loading of the test specimens

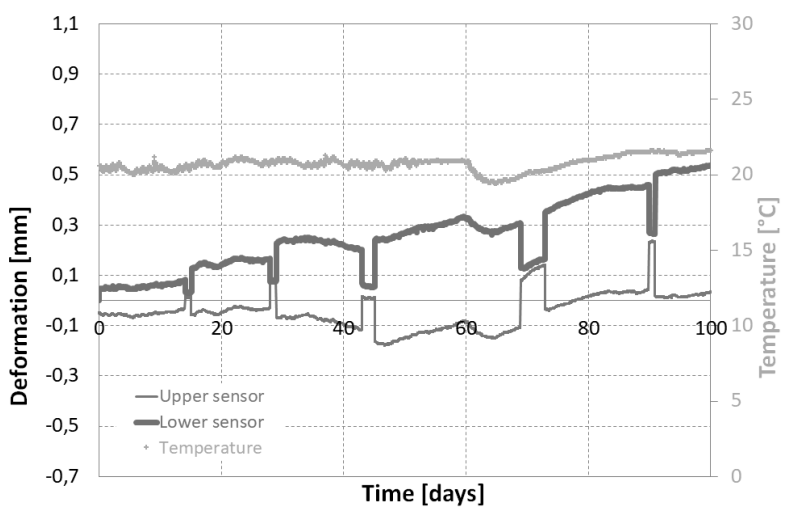

Fig. 6 Example of deformations of the measured sandwich composite structure (test specimen 7)

\subsection{Mechanical tests of the sandwich layers}

The theoretical models use material properties of each layer for evaluation of the deflection. Young's modulus of elasticity, shear modulus and Poisson's number belong among these properties. Because of its mechanical tests of the layer's materials were carried out.

The tensile test of epoxy-resin-impregnated glass laminate for skins was carried out. The tensile test was carried out parallel to the plain weave (direction $0^{\circ}$ ) and under angle $45^{\circ}$ to the plain weave (direction $45^{\circ}$ ). Five test specimens for each direction were tested. The length of the test specimen was $250 \mathrm{~mm}$, width $25 \mathrm{~mm}$ and thickness $0.8 \mathrm{~mm}$. The tensile test of epoxy-resin-impregnated glass laminate with plain weave was carried out according to Czech standard EN ISO 527-4 [18]. The tensile strength of epoxyresin-impregnated glass laminate with plain weave from experiments is $226 \mathrm{MPa}$ in direction $0^{\circ}$ and 62 $\mathrm{MPa}$ in direction $0^{\circ}$. Deformation of the laminate is $1.8 \%$ in direction $0^{\circ}$ and $3 \%$ in direction $45^{\circ}$. Young's modulus of the laminate is $13.9 \mathrm{GPa}$ in direction $0^{\circ}$ and $5.9 \mathrm{GPa}$ in direction $45^{\circ}$. The detailed results of tensile test of epoxy-resin-impregnated glass laminate with weight $600 \mathrm{~g} / \mathrm{m}^{2}$ with plain weave are described in [17]. Due to the composition of used epoxy-resin- impregnated glass laminate, laminate is considered as isotropic and homogenous material. 
The shear modulus was determined using Poisson's number and Young's modulus of elasticity.

The compression test of the sandwich core was carried out according to standard ASTM D 1621 [19]. Six test specimens were used for the compression test. The length of the test specimen was $150 \mathrm{~mm}$, width $20 \mathrm{~mm}$ and thickness $30 \mathrm{~mm}$. Dependence of the load on displacement is shown in Fig. 7.

The shear test according to standard ASTM C273 [20] was used for determination the core shear modulus. Four test specimens of the foam have been used for the shear test. The length of the test specimen was $240 \mathrm{~mm}$, width $50 \mathrm{~mm}$ and thickness $20 \mathrm{~mm}$. Dependence of the load on displacement is shown in Fig. 8. Experimentally obtained values correspond with values of mechanical properties obtained from manufacturer.

Other core material properties were used from the material list of the core Divinycell H100 obtained from manufacturer (DIAB GROUP) - Young's modulus of elasticity $130 \mathrm{MPa}$ and shear modulus $35 \mathrm{MPa}$ [21].

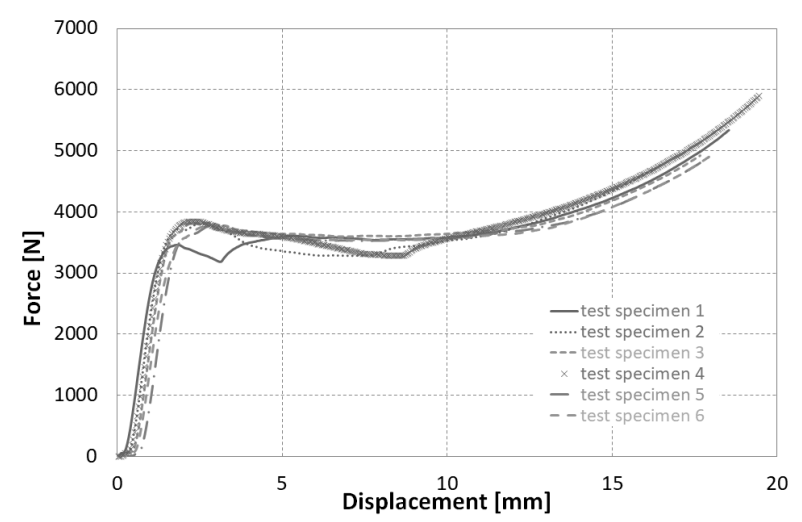

Fig. 7 Compression test of the sandwich core (light weight foam Divinycell H100)

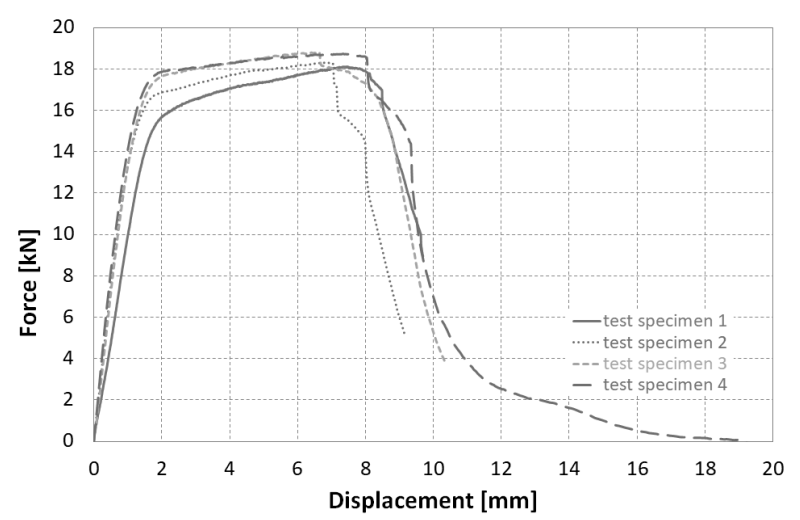

Fig. 8 Shear test of the sandwich core (light weight foam Divinycell H100)

\section{Results}

The three-point bending test was used for determining the deflection of the test specimen. The SOFO ${ }^{\circledR}$ SMARTape Compact deformation sensors with measuring length $600 \mathrm{~mm}$ were used for measuring the deformations of the tested sandwich beams. The sensors were placed between upper skin and sandwich core (upper sensor) and between lower skin and sandwich core (lower sensor). The SOFO ${ }^{\circledR}$ SMARTape Compact deformation sensors transform a strain variation into a change in the path unbalance between two optical fibres that are measured. The obtained values were used for evaluating the deflection. The deflection in the middle of the sandwich beam span was calculated from equation of the deflection line.

Test specimens were load by different ways (see Fig. 5). Test specimen 3 was repeatedly load by forces $12 \mathrm{~N}$ and $36 \mathrm{~N}$ during three months. The deflection was determined as average value obtained from repeated loading. Test specimen 4 was load with constant force $12 \mathrm{~N}$ and the deflection was determined as the average value of the deflections obtained during three months. Test specimen 5 was repeatedly load by forces $12 \mathrm{~N}$ and $60 \mathrm{~N}$ during three months. The deflection was determined as the average value obtained from repeated loading at force $60 \mathrm{~N}$. Test specimen 6 was load by forces $12 \mathrm{~N}, 36 \mathrm{~N}, 84 \mathrm{~N}, 108 \mathrm{~N}, 132 \mathrm{~N}$, $156 \mathrm{~N}$ and $180 \mathrm{~N}$. Test specimen 7 was load by forces $24 \mathrm{~N}, 48 \mathrm{~N}, 72 \mathrm{~N}$ and $96 \mathrm{~N}$. Each load was carried out for approx. two weeks. The deflection was determined for all values of the loading as average value of the deflection for given load. Obtained deflections for all test specimens are shown in Fig. 9. There are shown deflections obtained by upper and lower sensors.

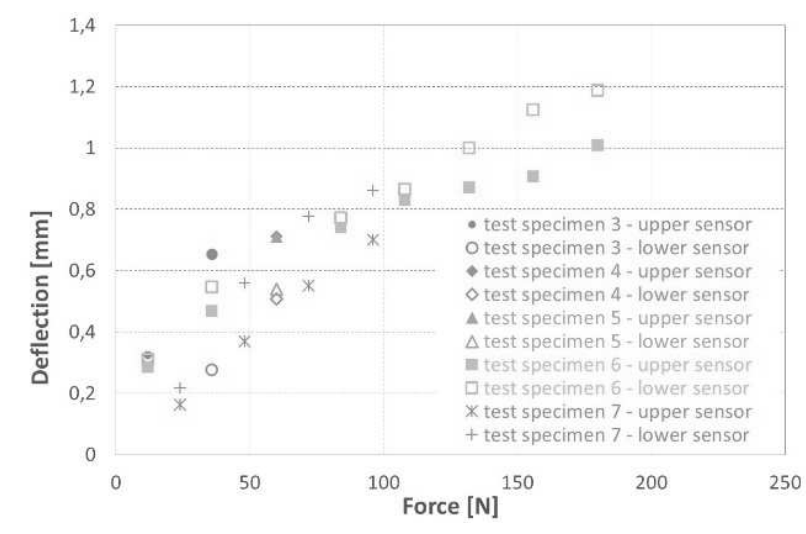

Fig. 9 Deflection of the test specimen - three-point bending test

\section{Discussion}

Experimentally obtained values of deflection were compared with theoretical models. The laminate theory with transverse shear, laminate theory without transverse shear, sandwich theory with transverse shear, sandwich theory without transverse shear was used for comparison with experiments. The Young's modulus of elasticity of the skin and Poisson's number of the skin were determined from the tensile test of the skin. Shear modulus of the skin was calculated 
from the definition of Poisson's number. Values of Young's modulus of elasticity of the core and shear modulus of the core were taken from DIAB GROUP [21]. The comparison of experimentally obtained deflection with theoretical models is shown in Fig. 10.

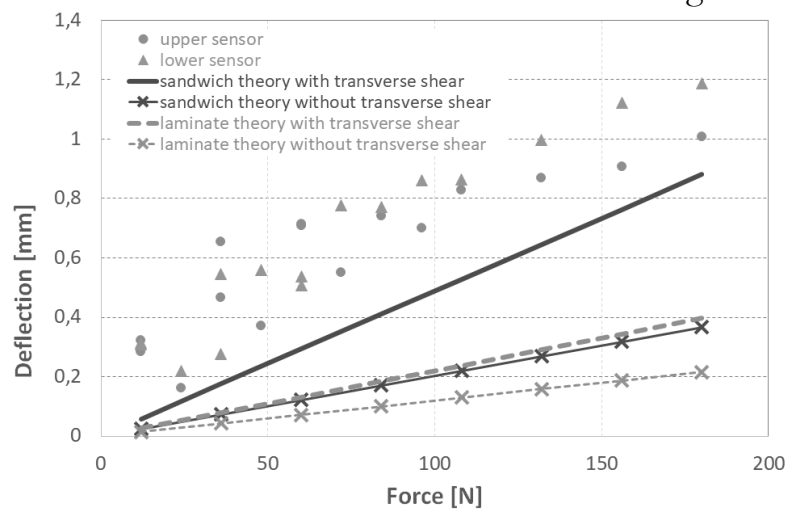

Fig. 10 Comparison of experimentally obtained deflection with theoretical models

It is evident from the comparison (see Fig. 10) that the sandwich theory without transverse shear, laminate theory with transverse shear and laminate theory without transverse shear are not suitable for this sandwich composite structure. The sandwich theory without transverse shear considers the thick core but it is not usable because it does not consider transverse shear. In real structure, transverse shear exists and the core carries it. The laminate theory with transverse shear considers that layers are thin, i.e. it does not consider that core is a thick layer. This model shows higher values of deflection than theoretical models without transverse shear due to this model considers transverse shear. According to the expectation the laminate theory without transverse shear shows the worst results. The reason of these results is that the model considers that each layer is thin and does not consider transverse shear.

Compared to other theories, the sandwich theory with transverse shear shows agreement with experiments. This result was expected due to assumptions of this theory. This theoretical model considers the core as homogenous thick layer and calculates with transverse shear. However, this theory shows differences between calculated values of deflection and experimentally obtained values of deflection. These differences are significant for higher values of the load. It is caused by the significantly plastic deformations of the core (see Fig. 7) while sandwich theory considers only elastic deformations.

\section{Conclusion}

- The experimental behaviour of the three-layered sandwich beam made of foam Divinycell H100 core sandwiched between epoxy-resinimpregnated glass laminates using embedded fibre-optic sensors is described in this paper.

- The three-point bending test was used for determining the sandwich beam deflection. The embedded fibre-optic sensors were used for measuring the deformations.

- The paper compared obtained deflections with theoretical models - sandwich theory with transverse shear, sandwich theory without transverse shear, laminate theory with transverse shear and laminate theory without transverse shear.

- Comparison of the experimental results with theoretical models (sandwich theory without transverse shear, laminate theory with transverse shear and laminate theory without transverse shear) shows that these theoretical models are not suitable for determination of the deflection of this type of the sandwich composite structure. These theories are simple and show third of the deflection compared to the experimentally obtained deflections.

- The comparison of experimentally obtained values of deflection with sandwich theory with transverse shear shows that this theory is suitable for this type of the sandwich composite structure.

- This theory is suitable for this sandwich structure because it considers that the core is thick and considers transverse shear which the core carries. However, there exist differences between values of deflections obtained experimentally and determined by the sandwich theory with transverse shear. It is caused by the significantly plastic deformations of the core while the sandwich theory considers only elastic deformations. The higher values of experimentally obtained deflections are caused due to core crushing.

- It means that the sandwich theory with transverse shear determines lower values of deflection. This must be considered in designing the sandwich composite structures.

\section{Acknowledgement}

This work has been supported by a research project of the Grant Agency of Czech Republic No. 14-35225P. 


\section{References}

[1] CARLSSON, L. A., KARDOMATEAS, G. (2011). A Structural and Failure Mechanics of Sandwich Composites. 386 pp. Springer-Verlag New York Inc. ISBN 1402032242

[2] KARLSSON, K. F., ÅSTRÖM, B. T. (1997). Manufacturing and applications of structural sandwich components. In: Composites Part A: Applied Science and Manufacturing, Vol. 28, Issue 2, pp. 97-111. ISSN 1359-835X

[3] KRZYŻAK, A., MAZUR, M., GAJEWSKI, M., DROZD, K., KOMOREK, A., PRZYBYŁEK, P. (2016) Sandwich Structured Composites for Aeronautics: Method of Manufacturing Affecting Some Mechanical Properties. In: International Journal of Aerospace Engineering, Vol. 2016, Article ID 7816912, 10 pp. ISSN 1687-5966

[4] RAZ, K., HORA, J., PAVLATA, P. (2017). Unconventional Materials Usage in Design of Vehicle Bodies. In: Manufacturing Technology, Vol. 17, No. 5. pp. 823-827. J.E. Purkyne University in Usti nad Labem, Czech Republic. ISSN 1213-2489

[5] ŽMINDÁK, M., NOVÁK, P., DEKÝŠ, V. (2017). Finite Element Analysis of the Delaminated Composite Plates Reinforced by Unidirectional Fibers. In: Manufacturing Technology, Vol. 17, No. 2. pp. 275-280. J.E. Purkyne University in Usti nad Labem, Czech Republic. ISSN 1213-2489

[6] DEMASI, L. (2008). $\propto^{3}$ Hierarchy plate theories for thick and thin composite plates: The generalized unified formulation. In: Composite Structures, Vol. 84, No. 3, pp. 256-270. ISSN: 0263-8223

[7] MANTARI, J. L., OKTEM, A. S., GUEDES SOARES, C. (2012). A new higher order shear deformation theory for sandwich and composite laminated plates. In: Composites Part B - Engineering, Vol. 43, No. 3, pp. 1489-1499. ISSN: 1359- 8368

[8] KARAMA, M., AFAQ, A. S., MISTOU, S. (2003). Mechanical behaviour of laminated composite beam by the new multi-layered laminated composite structures model with transverse shear stress continuity. In: International Journal of Solids and Structures, Vol. 40, No. 6, pp. 1525-1546. ISSN: 0020-7683

[9] DEMASI, L. (2009). $\propto^{6}$ Mixed plate theories based on the Generalized Unified Formulation. Part V: Results. In: Composite Structures, Vol. 88, No. 1, pp. 1-16. ISSN: 0263-8223
[10] AYDOGDU, M. (2009). A new shear deformation theory for laminated composite plates. In: Composite Structures, Vol. 89, No. 1, pp. 94101. ISSN: 0263-8223

[11] DEMASI, L. (2009). $\propto^{6}$ Mixed plate theories based on the Generalized Unified Formulation. Part I: Governing equations. In: Composite Structures, Vol. 87, No. 1, pp. 1-11. ISSN: 0263-8223

[12] BELBUTE, P. M. G. Study on the Bending Behavior of Sandwich Composite Beams https://fenix.tecnico.ulisboa.pt/downloadFile/395142126945/dissertaçao_resumo_alargado.pdf) - 11.6. 2019

[13] FANG, H., SHI, H., WANG, Y., Qi, Y., and LIU, W. (2016). Experimental and Theoretical Study of Sandwich Panels with steel Facesheets and GFRP Core. In: Advances in Materials Science and Engineering, Volume 2016, Article ID 7159205, $12 \mathrm{p}$.

[14] JIROUTOVÁ, D. (2016). Analysis of stress inside the sandwich composite structure. Advances and Trends in Engineering Sciences and Technologies II. 880 p. CRC Press. ISBN 9781315393827 (ebook)

[15] BERTHELOT, J., M. (1999). Composite Materials: Mechanical behavior and structural analysis. 646 p. New York: Springer. ISBN 0-387-98426-7

[16] Geotechnical \& Structural Instrumentation. Product Catalogue. http://www.edongil.com/contents/product/Roctest_modified.pdf) - 1. 7. 2019

[17] Jiroutova, D. (2016). Methodology of Experimental Analysis of Long-term Monitoring of Sandwich Composite Structure by Fibre-optic Strain Gauges. In: Manufacturing Technology, Vol. 16, No. 3. pp. 512-518. J.E. Purkyne University in Usti nad Labem, Czech Republic. ISSN 1213-2489

[18] EN ISO 527-4: Plastics - Determination of tensile properties - Part 4: Test conditions for isotropic and ortho-tropic fibre-reinforced plastic composites. Czech Standard. 1998, 20 p. Český normalizační institut, Prague

[19] ASTM D1621-16, Standard Test Method for Compressive Properties of Rigid Cellular Plastics, ASTM International, West Conshohocken, PA, 2016.

[20] ASTM C273 / C273M-16, Standard Test Method for Shear Properties of Sandwich Core Materials, ASTM International, West Conshohocken, PA, 2016

[21] www.diabgroup.com 